\title{
Computing H/D-Exchange Speeds of Single Residues from Data of Peptic Fragments
}

\author{
Ernst Althaus \\ MPI für Informatik \\ Saarbrücken, Germany \\ althaus@mpi-inf.mpg.de
}

\author{
Stefan Canzar ${ }^{*}$ \\ Université Henri Poincaré \\ LORIA, B.P. 239 \\ Vandœvre-lès-Nancy, France \\ scanzarempi-inf.mpg. de
}

\author{
Mark R. Emmett \\ Department of Chemistry, \\ Florida State University, \\ Tallahassee, FL, 32306
}

\author{
Andreas Karrenbauer \\ MPI für Informatik \\ Saarbrücken, Germany \\ karrenba@mpi-inf.mpg.de
}

\author{
Alan G. Marshall \\ Department of Chemistry, \\ Florida State University, \\ Tallahassee, FL, 32306 \\ marshall@magnet. fsu.edu
}

\section{Anke Meyer-Baese \\ Department of Electrical and \\ Computer Engineering \\ FAMU-FSU College of Engineering \\ Tallahassee, FL 32310-6046 \\ ambdeng. fsu.edu}

\author{
Huimin Zhang \\ Institute of Molecular \\ Biophysics, \\ Florida State University, \\ Tallahassee, FL, 323106 \\ hzhangemailer.sb.fsu.edu
}

\begin{abstract}
Determining the hydrogen-deuterium exchange speeds of single residues from data for peptic fragments obtained by FT-ICS MS is currently mainly done by manual interpretation. We provide an automated method based on combinatorial optimization. More precisely, we present an algorithm that enumerates all possible exchange speeds for single residues that explain the observed data of the peptic fragments.
\end{abstract}

\section{INTRODUCTION}

Hydrogen-deuterium exchange (HDX) is a chemical reaction in which a covalently bonded hydrogen atom is replaced by a deuterium atom, or vice versa. Usually the examined protons are on the amides in the backbone of a protein. The method gives information about the solvent accessibility of various parts of the molecule, and thus the tertiary structure of the protein.

In modern times HDX has primarily been monitored by the methods: NMR spectroscopy and mass spectrometry. Each of these

\footnotetext{
*Supported by the Centre National de Recherche Scientifique (CNRS) and by the German Academic Exchange Service (DAAD). ${ }^{\dagger}$ The work of the second author was done while he was visiting the Max-Planck-Institut für Informatik, Saarbrücken, Germany.
}

methods have their advantages and drawbacks. A major disadvantage of mass spectrometry is that one obtains exchange data for peptic fragments and assigning exchange rates to single residues has to be done by manual interpretation.

We provide an automated method to resolve this problem. More precisely, we present an algorithm that enumerates all possible exchange rates for single residues that explain the observed data of the peptic fragments. As the number of possibilities is often very large, we combine sets of assignments to equivalence classes which are easily interpreted such that the number of equivalence classes is typically very small.

The assignment of exchange rates to single residues from the data of the peptic fragments is a combinatorial problem. Hence, we applied methods from combinatorial optimization to it, i.e. we show how to formalize the problem into an integer linear program and propose a method to solve the problem.

The paper is organized as follows. In Section 2, we review the biochemical background of the underlying problem, motivating our research on this problem. Before giving an integer linear program for the combinatorial problem in Section 4, we make a formal description of the problem in Section 3. After that, we explain our solution method and the combination of possible solutions into equivalence classes in Section 5. In Section 6, we discuss the computational complexity of the problem and in Section 7, we show some experimental results of our algorithm. Finally, we give a short conclusion.

\section{BIOCHEMICAL BACKGROUND}

Determination of protein-protein interaction is best accomplished by X-ray crystal diffraction and NMR [9] because both methods provide the highest resolution of the sites of interaction. On the downside, both methods require large (milligram) quantities of protein. Other techniques rely on chemical or photo-induced reactions
Permission to make digital or hard copies of all or part of this work for personal or classroom use is granted without fee provided that copies are not made or distributed for profit or commercial advantage and that copies the full citation on the first page. To copy otherwise republish, to post on servers or to redistribute to lists, requires prior specific permission and/or a fee.

SAC'08 March 16-20, 2008, Fortaleza, Ceará, Brazil

Copyright 2008 ACM 978-1-59593-753-7/08/0003 ...\$5.00.
Konstanzer Online-Publikations-System (KOPS)

URN: http://nbn-resolving.de/urn:nbn:de:bsz:352-opus-122087

URL: http://kops.ub.uni-konstanz.de/volltexte/2010/12208 
with MS analysis [5, 3] to reveal functional groups that are exposed to the solvent. These methods also suffer from physical limitations.

Another method utilizes hydroxyl radical reactions with alkyl $\mathrm{C}-\mathrm{H}$ bonds. The $\mathrm{OH}$ tends to react mainly with surface-exposed residues providing a good footprint of the solvent exposed surface of the protein(s) $[2,6]$. The modification is covalent and thus irreversible, but each modification can potentially change the conformation of the protein, thus skewing results.

Exchange of labile hydrogens for deuteriums (HDX) as a probe of protein surface accessibility does not change the conformation of the protein. Advantages over NMR and X-ray crystallography structural determination are the ability to work at low concentration and high molecular weight.

The experiment is initiated by dilution of the protein solution into a biological buffer made with $D_{2} O$. Solvent accessible hydrogens are exchanged with deuterium. The exchange is quenched (greatly slowed) by dropping the $\mathrm{pH}$ to between $\mathrm{pH} 2.3$ and $\mathrm{pH} 2.5$ and lowering the temp to approximately $0^{\circ} \mathrm{C}$. The protein complex is digested with a protease that is active under quench conditions (such as pepsin) and on-line liquid chromatography is performed directly to the FT-ICR MS. Deuterium incorporation is monitored by the increase in mass of each peptic fragment as the deuteron is added.

The data sets produced are large and each spectrum has hundreds of overlapping peptic fragments. From this data, the exchange rate is easily determined for the same peptic fragments from the protein and the protein/protein complex. [4]. When peptic fragments are not directly comparable, but are overlapping (Figure 1) manual interpretation must be performed to assign exchange rate to single residues. HDX data analysis is the greatest bottle-neck in these experiments, thus automated data analysis is necessary.

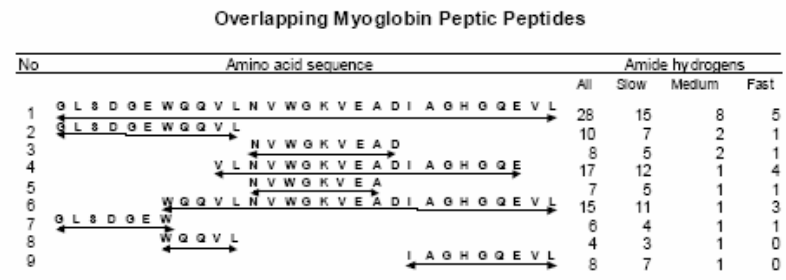

Figure 1: Overlap of peptic fragments as seen in an HDX of the model protein myoglobin. The table on the right shows the total number of amide hydrogens exchanged in each peptide and the number of amide hydrogens predicted to be either slow, medium or fast as predicted by maximum entropy method (MEM) evaluation of the H/D exchange rate distribution [8].

Furthermore, we are interested in all such assignments, as we want to determine protein conformation, protein/protein interaction, and protein/ligand interactions. This data will be useful in the design and synthesis of small molecules to be used as therapeutic agents.

\section{MATHEMATICAL ABSTRACTION}

In an idealized setting, we are considering the following problem. We are given a sequence $(1, \ldots, n)$ of residues, a set

$$
\mathcal{F} \subseteq\{(i, \ldots, j): 1 \leq i \leq j \leq n\}
$$

of peptic fragments, and a set of possible exchange rates $\mathcal{S}=$ $\{1, \ldots K\}$. We denote a fragment $(i, \ldots, j)$ by $(i, j)$ and refer to an exchange rate as a color. For each fragment $(i, j) \in \mathcal{F}$ and each color $k \in \mathcal{S}$, we are given the number $b_{(i, j)}^{k}$ of residues with color $k$ within the fragment $(i, j)$. The corresponding vector $b^{k}$ is referred to as the right hand side for color $k$. In our experimental data, we consider exactly three different colors (slow, medium, and fast), i.e. $K=3$.

We have to compute an assignment $\pi:\{1, \ldots, n\} \mapsto \mathcal{S}$ which assigns a color to each residue. This assignment has to respect our knowledge on the peptic fragments, i.e. $\pi:\{1, \ldots, n\} \mapsto \mathcal{S}$ such that $b_{(i, j)}^{k}=|\{i \leq l \leq j: \pi(l)=k\}|$ for all given fragments

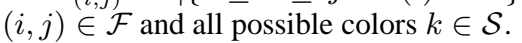

In real setting we will have errors in the data from our experiments. Hence we want to compute all assignments that minimize the total sum of errors, i.e. the assignments minimizing

$$
\sum_{k \in \mathcal{S}} \sum_{(i, j) \in \mathcal{F}} e_{(i, j)}^{k}
$$

where $e_{(i, j)}^{k}=\left|b_{(i, j)}^{k}-\right|\{i \leq l \leq j: \pi(l)=k\}||$.

\section{MATHEMATICAL MODEL}

First we formulate the idealized problem above as an integer linear program. More precisely, we show an integer linear program whose feasible solutions correspond to the feasible assignments of colors to residues.

Let $\pi:\{1, \ldots, n\} \mapsto \mathcal{S}$ be an assignment of colors to residues and for each $k \in \mathcal{S}$ let $x^{k} \in\{0,1\}^{n}$ be a set of binary variables and let $x=\left(x^{k}\right)_{k \in \mathcal{S}} \in\{0,1\}^{K n}$. Let furthermore $x_{i}^{k}=1$ if $\pi(i)=k$ and $x_{i}^{k}=0$ otherwise. Notice that all assignments satisfy $\sum_{k \in \mathcal{S}} x_{i}^{k}=1$ for all $i \in\{1, \ldots, n\}$ as every residue has exactly one color assigned. Furthermore every $\{0,1\}$-assignment to $x \in\{0,1\}^{K n}$ satisfying $\sum_{k \in \mathcal{S}} x_{i}^{k}=1$ for all $i \in\{1, \ldots, n\}$ corresponds to an assignment.

An $\{0,1\}$-assignment $x$ corresponds to a feasible $\pi$, iff furthermore $\sum_{l=i}^{j} x_{l}^{k}=b_{(i, j)}^{k}$ for all $(i, j) \in \mathcal{F}$ and $k \in \mathcal{S}$.

Assume now, we want to compute an assignment with minimum number of errors. Notice that we want to minimize a sum of absolute values. We use a standard trick to formulate such a problem as an integer linear program. Assume we have a variable $e_{i, j}^{k}$ for every color $k \in \mathcal{S}$ and every fragment $(i, j) \in \mathcal{F}$ whose value should be the error of the assignment $x$ for the color $k$ and the fragment $(i, j)$. Hence we want to minimize $\sum_{k \in \mathcal{S}} \sum_{(i, j) \in \mathcal{F}} e_{(i, j)}^{k}$. It suffices to formulate conditions on $e_{(i, j)}^{k}$ that enforce it to be at least the error. As we minimize, the value will not be larger than the error in an optimal solution. To enforce $e_{(i, j)}^{k}$ to be at least the error, the two linear constraints $e_{(i, j)}^{k} \geq \sum_{l=i}^{j} x_{l}^{k}-b_{(i, j)}^{k}$ and $e_{(i, j)}^{k} \geq-\sum_{l=i}^{j} x_{l}^{k}+b_{(i, j)}^{k}$ are sufficient.

Hence the integer linear program, we are looking at is

$$
\begin{array}{lll}
\min & \sum_{k \in \mathcal{S}} \sum_{(i, j) \in \mathcal{F}} e_{(i, j)}^{k} & \\
\text { s.t. } & e_{(i, j)}^{k} \geq \sum_{l=i}^{j} x_{l}^{k}-b_{(i, j)}^{k} & \text { for all } k \in \mathcal{S},(i, j) \in \mathcal{F} \\
& e_{(i, j)}^{k} \geq-\sum_{l=i}^{j} x_{l}^{k}+b_{(i, j)}^{k} & \text { for all } k \in \mathcal{S},(i, j) \in \mathcal{F} \\
& \sum_{k \in \mathcal{S}} x_{l}^{k}=1 & \text { for all } 1 \leq l \leq n \\
& x \in\{0,1\}^{K n} &
\end{array}
$$

We refer to this integer linear program as basic-ILP.

\section{SOLUTION OF THE MATHEMATICAL MODEL}

We implemented our approach using the C++-Library $\mathrm{SCIL}^{1}$ to

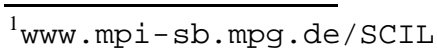


solve integer linear programs. SCIL uses the libraries LEDA $^{2}$ and SCIP $^{3}$. SCIP uses CPLEX ${ }^{4}$ or SoPlex ${ }^{5}$ as solver for linear programs. The underlying solution method is branch-\&-bound, which is described in detail in [7].

In order to find all solutions within a given error bound $e$, we add the constraint $\sum_{k \in \mathcal{S}} \sum_{(i, j) \in \mathcal{F}} e_{(i, j)}^{k} \leq e$ to the integer linear program and hence are faced with the problem of computing all feasible solutions of an integer linear program. We do this with a branching-approach similar to the branch-\&-bound approach described above. We solve the linear relaxation. If the linear relaxation if infeasible, we stop the search on this branch. If the solution is integral, we store it (if we haven't found this solution yet). If there is a binary variable which is not fixed so far (i.e. not set to 0 or 1 ), we pick one such variable $x_{l}^{k}$ and solve the two subproblems where we fix the variable to 0 or 1 recursively. Notice that it is possible that we branch on a variable which already has an integral value. In this case, the solution of the linear relaxation of the subproblem will be the same as in problem itself. Nevertheless, we will terminate, as there are only a finite number of variables to branch on.

In our experiments, it turns out that finding a single solution is very fast, whereas finding all solutions takes quite some time (see Table 1 in Section 7). The reason is mainly that the number of solutions is quite large. This is as there are quite large intervals such that no fragment starts or ends within an interval. Let $\mathcal{P}$ be the partition of $\{1, \ldots, n\}$ into a minimal number of intervals, such that for each element of $p \in \mathcal{P}$ and each fragment $f \in F$ either $p \subseteq f$ or $p \cap f=\emptyset$. Notice that for an assignment $\pi$, we can get further assignments with the same total error, if we permute the colors within these intervals, i.e. if $i, j \in p$ for $p \in \mathcal{P}$ and $\pi$ is a feasible assignment than $\pi^{\prime}$ with $\pi^{\prime}(i)=\pi(j), \pi^{\prime}(j)=\pi(i)$ and $\pi^{\prime}(l)=\pi(l)$ for $l \neq i, j$ is a feasible assignment. We call two assignments equivalent, if one can be obtained by the other by iteratively applying this rule.

Hence we modify our integer linear program in order to enumerate equivalent solutions only once. For $k \in \mathcal{S}$ and $p \in \mathcal{P}$, we replace the binary variables $\left(x_{l}^{k}\right)_{l \in p}$ by a single integer variable $y_{p}^{k}$ with $y_{p}^{k}:=\sum_{l \in p} x_{l}^{k}$. Moreover, let $A$ be the $|\mathcal{F}| \times|\mathcal{P}|$ matrix, i.e. for every $f \in \mathcal{F}$ and $p \in \mathcal{P}$, the corresponding entry is given by

$$
a_{f, p}= \begin{cases}1 & \text { if } p \subseteq f \\ 0 & \text { otherwise }\end{cases}
$$

In matrix notation the constraints are then of the form

$$
\begin{gathered}
-A y^{k}+e^{k} \geq-b^{k} \\
A y^{k}+e^{k} \geq b^{k}
\end{gathered}
$$

for all $k \in \mathcal{S}$. Hence our integer linear program gets

$$
\begin{array}{lll}
\min & \sum_{k \in \mathcal{S}} \sum_{f \in \mathcal{F}} e_{f}^{k} & \\
\text { s.t. } & -A y^{k}+e^{k} \geq-b^{k} & \text { for all } k \in \mathcal{S} \\
& A y^{k}+e^{k} \geq b^{k} & \text { for all } k \in \mathcal{S} \\
& \sum_{k \in \mathcal{S}} y^{k}=P & \\
& y \geq 0, \text { integer } &
\end{array}
$$

\footnotetext{
${ }^{2}$ www.mpi-sb.mpg.de/LEDA/

${ }^{3}$ www.zib.de/bib/pub/pw

${ }^{4}$ www. $i \log$. com

${ }^{5}$ www.zib.de/Optimization/Software/Soplex
}

where $P$ is the vector that contains $|p|$ for each component $p \in \mathcal{P}$.

We refer to this integer linear program as improved-ILP. We compute all solutions within a certain error bound by following basically the same approach as described above. The number of solutions is just a fraction of the number of solutions of the original integer linear program (see Section 7).

\section{COMPUTATIONAL COMPLEXITY}

We first consider the case with two colors. That is, we have the constraints $y_{p}^{1}+y_{p}^{2}=|p|$ for all $p \in \mathcal{P}$. This allows us to simplify the linear program considerably. We replace $y_{p}^{2}=|p|-y_{p}^{1}$ and omit the superscript of the $y$-variables in the following. This yields

$$
\begin{array}{rlrl}
-A y+e^{1} & \geq-b^{1} & A y+e^{2} & \geq F-b^{2} \\
A y+e^{1} & \geq b^{1} & -A y+e^{2} & \geq-F+b^{2}
\end{array}
$$

where $F$ is the vector of fragment sizes. We may get rid of half of the constraints by the following observation. Let $b:=\max \left\{b^{1}, F-\right.$ $\left.b^{2}\right\}$ and $\bar{b}:=\min \left\{b^{1}, F+b^{2}\right\}$ where the maximum is taken component-wise. Let $y$ be an arbitrary feasible solution with minimum total error $\sum_{f \in \mathcal{F}} e_{f}^{1}+e_{f}^{2}$. We may consider the contribution of each fragment independently for that particular $y$. We may rename the error variables $e^{1}$ and $e^{2}$ component-wise according to $b$ and $\bar{b}$, i.e.

$$
e_{f}:=\left\{\begin{array}{ll}
e_{f}^{1} & \text { if } b_{f}=b_{f}^{1} \\
e_{f}^{2} & \text { otherwise }
\end{array} \quad \bar{e}_{f}:= \begin{cases}e_{f}^{1} & \text { if } \bar{b}_{f}=b_{f}^{1} \\
e_{f}^{2} & \text { otherwise }\end{cases}\right.
$$

For each $f \in \mathcal{F}$ with $\bar{b}_{f} \leq a_{f}^{T} y \leq b_{f}$, we have $e_{f}^{1}+e_{f}^{2}=b_{f}-\bar{b}_{f}$. If $a_{f}^{T} y>b_{f}$, we get $e_{f}^{1}+e_{f}^{2}=2 e_{f}+b_{f}-\bar{b}_{f}$. Analogously, we get $e_{f}^{1}+e_{f}^{2}=2 \bar{e}_{f}+b_{f}-\bar{b}_{f}$ if $a_{f}^{T} y<\bar{b}_{f}$. Hence, it is sufficient to optimize the following linear program

$$
\begin{array}{ll}
\min & \sum_{f \in \mathcal{F}} e_{f}+\bar{e}_{f} \\
\text { s.t. } & -A y+e \geq-b \\
& A y+\bar{e} \geq \bar{b} \\
& -y \geq-P \\
& y, e, \bar{e} \geq 0
\end{array}
$$

which is integral if $b$ and $\bar{b}$ are integral since the constraint matrix is totally unimodular. The corresponding dual LP is given by

$$
\begin{array}{ll}
\max & -b^{T} f^{1}+\bar{b}^{T} f^{2}-P^{T} f^{3} \\
\text { s.t. } & -A^{T} f^{1}+A^{T} f^{2}-f^{3} \leq 0 \\
& 0 \leq f^{1,2} \leq 1 \\
& 0 \leq f^{3}
\end{array}
$$

which is equivalent to (multiplying the objective function by -1 and introducing slack variables)

$$
\begin{aligned}
-\min & b^{T} f^{1}-\bar{b}^{T} f^{2}+P^{T} f^{3} \\
\text { s.t. } & -A^{T} f^{1}+A^{T} f^{2}-f^{3}+f^{4}=0 \\
& 0 \leq f^{1,2} \leq 1 \\
& 0 \leq f^{3,4}
\end{aligned}
$$

We will show next that this LP is a Minimum Cost Circulation Problem. To this end, let $M$ be the matrix of the equality constraints, i.e.

$$
M:=\left(\begin{array}{llll}
-A^{T} & A^{T} & -I & I
\end{array}\right)
$$




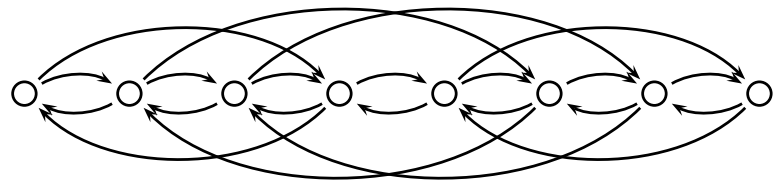

Figure 2: Example of a fragment graph with $|P|=7$. The corresponding fragments are $(1,3),(2,5),(3,6)$, and $(5,7)$.

Note that this matrix has the column-wise consecutive-ones property. By row operations like in Gaussian elimination, we can easily transform $M$ such that each column contains exactly one 1 and one -1 , as follows. We add the dummy constraint $0=0$ at the end and subtract from each row its predecessor. The resulting matrix, say $\bar{M}$, can be considered as the node-arc-incidence matrix of a directed graph. Since the right hand side remains unchanged, we get a Minimum Cost Circulation problem on a graph with $|\mathcal{P}|+1$ nodes and $O(|\mathcal{P}|+|\mathcal{F}|)$ arcs [1]. As a matter of fact, we have for each variable $y_{p}$ two arcs corresponding to the constraint $0 \leq y_{p} \leq|p|$ and for each fragment $(i, j)$ the $\operatorname{arcs}(i, j+1)$ and $(j+1, i)$ as depicted in Figure 2.

We may use any algorithm that solves the Minimum Cost Circulation problem, e.g. Cycle Canceling or Successive Shortest Path (see [1] for further reference). Both approaches have their advantages. The former always maintains a feasible circulation, i.e. we start with the zero flow and augment flow along negative cycles in the residual network until no negative cycle remains. Since the residual network with respect to an optimal circulation does not contain a directed negative circuit, we can find node potentials, i.e. a corresponding dual solution, using the Bellman-Ford algorithm in $O(|\mathcal{P}| \cdot|\mathcal{F}|)$ time. The difference between the potential of two neighboring nodes then yields the value of the corresponding $y$-variable. The errors are determined straight forward. If there is a solution without error this approach yields a solution within the running time of Bellman-Ford. On the other hand, the Successive Shortest Path algorithm maintains similar node potentials such that the arc-weights remain non-negative. Since the total excess is bounded by $|\mathcal{P}|$ in our case, the running time of that algorithm is $O\left(|\mathcal{P}| \cdot|\mathcal{F}|+|\mathcal{P}|^{2} \log |\mathcal{P}|\right)$.

For three or more colors the complexity is open. The totally unimodularity of the constraint matrix is destroyed, i.e. there are instances with fractional vertices, e.g. the one from Figure 2 with the appropriate right hand sides. Moreover, there is an instance which has a positive error, but the value of the LP is 0 . Hence the integrality gap is infinite. If the number of colors is not fixed but part of the input, the problem is NP-complete.

\section{EXPERIMENTS}

We applied our branch-\&-bound solution method to several real instances and to randomly generated instances, as we only have a limited number of real instances at hand.

The real instances had between 28 and 57 residues and between 16 and 50 fragments. The solutions with a minimal number of errors could be computed in less than 0.1 seconds for all instances. All (non-equivalent) solutions with a minimal number of errors, between 6 and 62 in number, could be computed in less than 5 seconds, where the running time greatly depends on the number of solutions (see Table 1). Computing all solutions using the basicILP takes much longer as with the improved-ILP.

The results for the real instances are very promising as the small number of easily interpretable classes of equivalent solutions can be

\begin{tabular}{|c|c|c|c|c|c|c|c|c|}
\hline \multicolumn{3}{|c|}{ Instance } & \multicolumn{3}{|c|}{ Basic-ILP } & \multicolumn{3}{|c|}{ Improved-ILP } \\
\hline$n$ & $|\mathcal{F}|$ & $|\mathcal{P}|$ & $\mathrm{T}$ (One) & $\mathrm{T}(\mathrm{All})$ & \#-Sol & $\mathrm{T}$ (One) & T(All & \#-Sc \\
\hline & 16 & 121 & 0.02 & 418 & 1026 & 0.02 & & \\
\hline & 34 & & & & & & & \\
\hline & 50 & 373 & & & & & & \\
\hline 37 & 17 & 16 & 0.03 & 2084.12 & 4529151 & 0.01 & 0.2 & \\
\hline
\end{tabular}

Table 1: We give the characteristics of the instance, i.e. the number of residues $(n)$, the number of fragments $(\mathcal{F})$, number of intervals $(\mathcal{P})$, and the minimal error of an solution $(\epsilon)$. For the basic and the improved ILP formulation, we give the solution times in seconds and the number of solutions found.

used in protein structure prediction tools and for manual inspection.

To evaluate the running time of our approach more closely, we created the random instances as follows. We generated a sequence of a given number of residues. For each residue, we randomly chose a color with a biased coin. We have chosen a probability of 0.6 for slow, and 0.2 for medium and fast, which reflect approximately the numbers we observed in the real instances. Then we generated random fragments, i.e. we chose $i, j \in\{1, \ldots, n\}$ with $i<j$ at random and repeated this $n / 2$ times. In our experiments, we use $n=50,100,150,200,250,300,500,1000$. Note that the actual numbers of variables for the Improved-ILP is lower than $n$. However, it is only a slight difference due to our random choice of the fragments. We computed for each fragment the numbers of residues having a certain color and added a random Gaussian noise on these numbers to reflect the errors in the measurement. Since those artificial instances are generated by a rather simple model of real measurements, we have to be careful about a quantitative analysis. We generated one series of instances without noise and three further series with gaussian noise of mean 0 and different standard deviations.

First, we evaluated the running times to find one optimal solution (see Figure 3). As one can see, the noise has a growing effect the more variables we consider. At a first glance, this log-log-plot suggests a power law behavior. Hence, we fitted a power function for each series. For the sake of illustration, we only show the straight line representing the best fit of a power function to running times of the instances without noise. However, it demonstrates that the measurement points tend to follow a slight curvature to the left. Moreover, the exponent of this fit is roughly 2.1 and for the other series it tends towards 3 with growing noise. Though integer linear programming is exponential in general, this is appearantly not the case in the considered range $n \leq 1000$. It seems that the running times are dominated by solving linear programs. Since this involves solving systems of equations, we assume a cubic polynomial for a further fit of the running times. In fact, the curve that one can see in Figure 3, which corresponds to the measurements with the most noise, nicely fits the data.

The effect of the noise on the running times becomes more apparant when we enumerate all optimal solutions (see Figure 4). One reason for this is that the higher the minimum error the more optimal solutions exist at this value. Moreover, the number of optimal solutions also grows with increasing number of variables. The straight lines in the log-log-plot show fits of power functions with exponents ranging from 3.2 to 4.3 roughly. However, the distribution of the running times is too broad to get a significant result with the limited number of measurements.

Anyways, it would be more interesting to have more real world instances than the randomly generated ones to evaluate our method and future work such as generalizing the combinatorial approach 


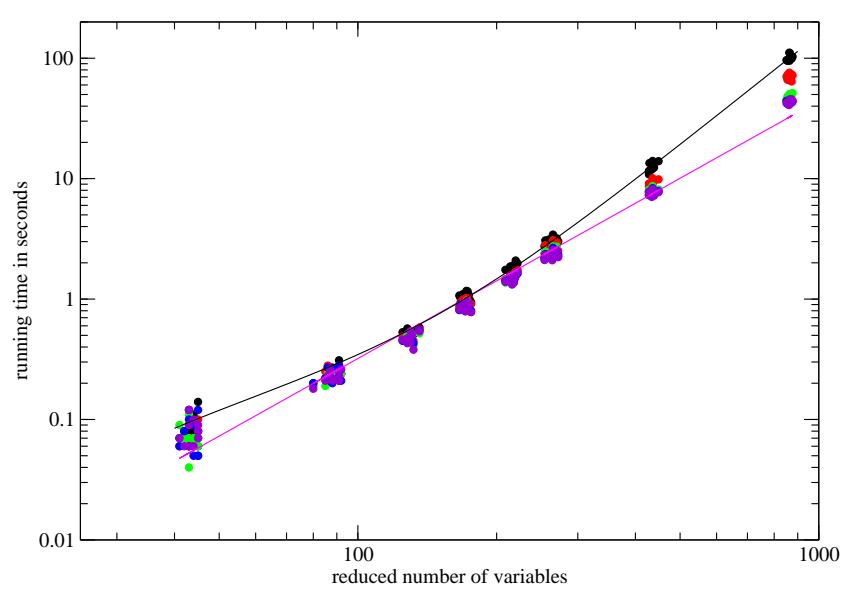

Figure 3: Running time of the Improved-ILP approach.

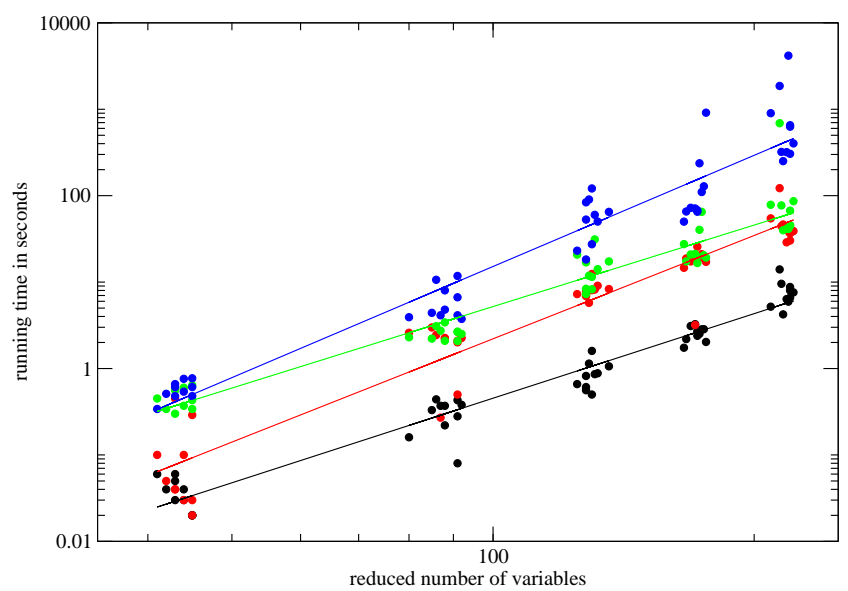

Figure 4: Time to enumerate all solutions with minimum error. The lowest of the four straight lines corresponds to the measurements without noise.

to more than two colors. However, gathering the experimental data involves a considerable effort. For the sake of completeness, we briefly explain the applied techniques in the following.

The entire HDX experiment was automated with a LEAP robot (HTS PAL, Leap Technologies, Carrboro, NC). Automation of the experiment reduces human error and reduces deuterium for hydrogen back-exchange. All time points where interlaced and performed in triplicate to ensure experimental reproduceability. After digestion, the protein digest was injected from a $10 \mu \mathrm{L}$ loop to either a $1 \mathrm{~mm}$ x $50 \mathrm{~mm}$ C5 column (Phenomenex) or a Pro-Zap Prosphere HP C18 HR 1.5u $10 \mathrm{~mm}$ x $2.1 \mathrm{~mm}$ (Alltech). A rapid gradient $2 \%$ B to $95 \%$ B in 1.5 min (A: acetonitrile/H2O/formic acid 5/94.5/0.5, B: acetonitrile/H2O/formic acid 95/4.5/0.5) was used to elute peptides. The eluent was post-column split and infused by microelectrospray ionization into a custom built 14.5 T LTQ FTICR mass spectrometer. Data was analyzed by an in-house analysis package (Sasa 2007 paper, submitted).

\section{CONCLUSION}

We proposed an approach to assign exchange rates to single residues from data of peptic fragments based on integer linear programming. The resulting algorithm is very efficient. Furthermore we gave a combinatorial algorithm for the case of two exchange speeds.

In the case of three or more different exchange speeds, the complexity of the problem remains open. Furthermore, we want to extend the combinatorial approach for three exchange speeds and so that it is able to enumerate all solutions even faster, which could be important as the sizes of the problems will probably increase in the future.

\section{Acknowledgements}

Work supported by NSF (DMR-00-84173), NIH (GM 78359), Florida State University, and the National High Magnetic Field Laboratory in Tallahassee, FL.

\section{REFERENCES}

[1] R. K. Ahuja, T. L. Magnanti, and J. B. Orlin. Network flows: theory, algorithms, and applications. Prentice Hall Inc., Englewood Cliffs, NJ, 1993.

[2] M. Goshe, Y. Chen, and V. Anderson. Identification of the sites of hydroxyl radical reaction with peptides by hydrogen/deuterium exchange: Pr evalence of reactions with the side chains. Biochemistry, 39(7):1761-1770, 2000.

[3] S. Kang, A. Hawkridge, K. Johnson, D. Muddiman, and P. Prevelige. Identification of subunit-subunit interactions in bacteriophage p22 procapsids by chemical cross-linking and mass spectrometry. Journal of Proteome Research, 5(2):370-377, 2006.

[4] T. Lam, J. Lanman, M. Emmett, C. Hendrickson, M. A.G., and P. Prevelige. Mapping of protein:protein contact surfaces by hydrogen/deuterium exchange, followed by on-line high-performance liquid chromatography-electrospray ionization fourier-transform ion-cyclotron-resonance mass analysis. Journal of Chromatography A, 982(1):85-95, 2002.

[5] J. Leite and M. Cascio. Probing the topology of the glycine receptor by chemical modification coupled to mass spectrometry. Biochemistry, 41(19):6140-6148, 2002.

[6] J. Sharp, J. Becker, and R. Hettich. Analysis of protein solvent accessible surfaces by photochemical oxidation and mass spectrometry. Analytical Chemistry, 76(3):672-683, 2004.

[7] L. A. Wolsey. Integer programming. Wiley-interscience series in discrete mathematics and optimization. Wiley \& Sons, New York, 1998.

[8] Z. Zhang, S. Guan, and A. G. Marshall. Enhancement of the Effective Resolution of Mass Spectra of High Mass Biomolecules by Maximum Entropy-Based Deconvolution to Eliminate the Isotopic Natural Abundance Distribution. Journal of American Society of Mass Spectrometry, 8:659 670, 1997.

[9] E. Zuiderweg. Mapping protein-protein interactions in solution by nmr spectroscopy. Biochemistry, 41(1):1-7, 2002. 